\title{
Plonski's (metric) yield tables formulated
}

\author{
by Bijan Payandeh
}

\begin{abstract}
A four-parameter sigmoidal growth model was employed to express various characteristics of interest in Plonski's metric yield tables as functions of stand age and site index. Except in a few cases, excellent fits were obtained. The resulting equations will facilitate direct computer use of Plonski's metric yield tables.
\end{abstract}

\section{Résumé}

Un modèle de croissance sigmoïdale à quatre paramètres a été utilisé pour exprimer diverses caractéristiques intéressantes des tables métriques de rendement de Plonski, en fonction de l'âge du peuplement et de la qualité du site. A l'exception de quelques cas, une adéquation excellente ont été obtenue. Les équations qui en résultent faciliteront l'utilisation directe par l'ordinateur des tables métriques de rendement de Plonski.

\section{Introduction}

Plonski $(1956,1960,1974)$ constructed normal yield tables for the major forest types in Ontario: black spruce (Picea mariana [Mill.] B.S.P.), jack pine (Pinus banksiana Lamb.), aspen (Populus tremuloides Michx.), white birch (Betula papyrifera Marsh.), red pine (Pinus resinosa Ait.), white pine (Pinus strobus L.), and tolerant hardwoods. Plonski (1956) gave a detailed account of the construction and use of his tables.

Despite their shortcomings, normal yield tables have been used extensively in forest-management and economicevaluation research and as a basis for management-planning inventories. This is particularly true of Plonski's yield tables in Ontario. However, these tables were constructed on the basis of balanced and harmonized curves; i.e., no equations were given for them. Thus, to utilize the tables, it is necessary to look up or interpolate values for given variables individually from the appropriate tables or graphs. Payandeh (1973) formulated Plonski's Imperial yield tables by means of stepwise multiple linear regression analysis. The purpose of this note is to present similar information for Plonski's metric yield tables so as to facilitate computer processing and to expedite interpolation between table values.

\section{Methods}

For each species, values for various characteristics of interest, such as stand age, height, basal area, diameter, number of trees, gross total volume, etc., were read directly from the appropriate tables. A four-parameter nonlinear model was used to express each stand characteristic of interest as a function of stand age and site index:

$$
Y=B_{1} S_{2}^{\mathrm{B}}\left(1-e^{\mathrm{B}}{ }_{3}^{\mathrm{A}}\right)^{\mathrm{B}}{ }_{4}+E
$$

where: $Y=\mathrm{a}$ characteristic of interest such as stand height, average diameter, basal area, number of trees, total gross volume, etc.
$S=$ site index (m) at index age 50 years,

$A=$ stand age (years),

$e=$ base of natural logarithms

$B_{1}, B_{2}, B_{3}, B_{4}=$ model parameters to be estimated,

$E=$ error term of the model

\section{Results}

The estimated parameters of the above model for the various characteristics of interest for the seven species are summarized in (Table 1). With a few exceptions (the $R^{2}$ values for basal area per hectare for all species except for aspen and white birch, and the number of trees per hectare for aspen were between 0.92 and 0.96 ), these equations provide very good approximations $\left(R^{2} \geq 0.98\right)$ of the tabular values.

\section{References}

Payandeh, B . 1973. Plonski's yield tables formulated. Dept. Environ., Can. For. Serv., Ottawa, Ont. Publ. NO. 1318. $14 \mathrm{p}$.

Plonski, W.L. 1956. Normal yield tables for black spruce, jack pine, aspen and white birch in northern Ontario. Ont. Dep. Lands For., Timber Manage. Dir., Toronto, Ont. Rep. No. 24. 40 p.

Plonski, W.L. 1960. Normal yield tables for black spruce, jack pine, aspen, white birch, tolerant hardwoods, white pine and red pine for Ontario. Ont. Dep. Lands For., Toronto, Ont. Silvic. Ser. Bull. 2. 39 p.

Plonski, W.L. 1974. Normal yield tables (metric) for major forest species of Ontario. Ont. Min. Nat. Resour., Toronto, Ont. $40 \mathrm{p}$.

Forestry Canada, Ontario Region, P.O. Box 490, Sault Ste. Marie, Ontario P6A 5M7. 
Table 1. Estimated parameters for various characteristics of interest for the seven species in Plonski's (metric) yield tables

\begin{tabular}{|c|c|c|c|c|}
\hline Characteristic of interest & $\mathbf{B}_{1}$ & $\mathrm{~B}_{2}$ & $\mathbf{B}_{3}$ & $\mathbf{B}_{4}$ \\
\hline & \multicolumn{4}{|c|}{ Black spruce } \\
\hline Height (m) & 4.294 & 0.670 & -0.021 & 1.762 \\
\hline $\mathrm{DBH}(\mathrm{cm})$ & 4.926 & 0.604 & -0.019 & 1.632 \\
\hline No. trees/ha & 6100.858 & -0.695 & -0.009 & -1.208 \\
\hline $\mathrm{BA}\left(\mathrm{m}^{2}\right)$ & 18.009 & 0.369 & -0.033 & 2.452 \\
\hline Gross vol. $\left(\mathrm{m}^{3}\right)$, main stand & 51.472 & 0.830 & -0.027 & 3.413 \\
\hline Gross vol. $\left(\mathrm{m}^{3}\right)$, total production & 53.025 & 0.925 & -0.024 & 3.196 \\
\hline Gross merch. vol. $\left(\mathrm{m}^{3}\right)$ & 12.895 & 1.377 & -0.030 & 8.050 \\
\hline \multirow[t]{2}{*}{ Merch. vol. $\left(\mathrm{m}^{3}\right)$ to $7-\mathrm{cm} \mathrm{DBH}$} & 31.082 & 1.004 & -0.032 & 5.976 \\
\hline & \multicolumn{4}{|c|}{ Jack pine } \\
\hline Height & 1.393 & 0.980 & -0.039 & 1.794 \\
\hline $\mathrm{DBH}(\mathrm{cm})$ & 1.347 & 1.003 & -0.037 & 2.274 \\
\hline No. trees/ha & 17415.646 & -1.259 & -0.021 & -2.490 \\
\hline $\mathrm{BA}\left(\mathrm{m}^{2}\right)$ & 5.050 & 0.596 & -0.071 & 1.511 \\
\hline Gross vol. $\left(\mathrm{m}^{3}\right)$, main stand & 4.555 & 1.437 & -0.045 & 2.455 \\
\hline Gross vol. $\left(\mathrm{m}^{3}\right)$, total production & 7.065 & 1.429 & -0.039 & 2.767 \\
\hline Gross merch. vol. $\left(\mathrm{m}^{3}\right)$ & 1.984 & 1.690 & -0.062 & 8.070 \\
\hline \multirow[t]{2}{*}{ Merch. vol. $\left(\mathrm{m}^{3}\right)$ to $7-\mathrm{cm} \mathrm{DBH}$} & 2.563 & 1.611 & -0.068 & 8.015 \\
\hline & \multicolumn{4}{|c|}{ Aspen } \\
\hline Height & 1.719 & 0.928 & -0.033 & 1.516 \\
\hline $\mathrm{DBH}(\mathrm{cm})$ & 2.181 & 0.984 & -0.013 & 1.184 \\
\hline No. trees/ha & 48549.530 & -1.383 & -0.039 & -2.88 \\
\hline $\mathrm{BA}\left(\mathrm{m}^{2}\right)$ & 6.856 & 0.556 & -0.035 & 1.35 \\
\hline Gross vol. $\left(\mathrm{m}^{3}\right)$, main stand & 7.655 & 1.323 & -0.042 & 3.422 \\
\hline Gross vol. $\left(\mathrm{m}^{3}\right)$, total production & 6.962 & 1.446 & -0.038 & 3.453 \\
\hline Gross merch. vol. $\left(\mathrm{m}^{3}\right)$ & 5.043 & 1.412 & -0.057 & 8.127 \\
\hline \multirow[t]{2}{*}{ Merch. vol. $\left(\mathrm{m}^{3}\right)$ to $7-\mathrm{cm} \mathrm{DBH}$} & 5.647 & 1.408 & -0.047 & 4.606 \\
\hline & \multicolumn{4}{|c|}{ White birch } \\
\hline Height & 1.035 & 1.068 & -0.051 & 2.752 \\
\hline $\mathrm{DBH}(\mathrm{cm})$ & 0.418 & 1.414 & -0.035 & 2.447 \\
\hline No. trees/ha & 54382.856 & -1.568 & -0.025 & -2.459 \\
\hline $\mathrm{BA}\left(\mathrm{m}^{2}\right)$ & 1.987 & 0.919 & -0.066 & 3.746 \\
\hline Gross vol. $\left(\mathrm{m}^{3}\right)$, main stand & 2.476 & 1.604 & -0.059 & 5.293 \\
\hline Gross vol. $\left(\mathrm{m}^{3}\right)$, total production & 2.811 & 1.646 & -0.053 & 5.106 \\
\hline Gross merch. vol. $\left(\mathrm{m}^{3}\right)$ & 0.137 & 2.534 & -0.067 & 12.352 \\
\hline \multirow[t]{2}{*}{ Merch. vol. $\left(\mathrm{m}^{3}\right)$ to $7-\mathrm{cm}$ DBH } & 0.535 & 2.109 & -0.070 & 12.424 \\
\hline & \multicolumn{4}{|c|}{ Tolerant hardwoods } \\
\hline Height & 2.780 & 0.801 & -0.030 & 1.914 \\
\hline $\mathrm{BA}\left(\mathrm{m}^{2}\right)$ & 6.231 & 0.646 & -0.038 & 3.135 \\
\hline Gross vol. $\left(\mathrm{m}^{3}\right)$, main stand & 20.129 & 1.103 & -0.020 & 2.401 \\
\hline \multirow[t]{2}{*}{ Gross merch. vol. $\left(\mathrm{m}^{3}\right)$} & 11.719 & 1.191 & -0.027 & 4.165 \\
\hline & \multicolumn{4}{|c|}{ White pine } \\
\hline Height (m) & 4.881 & 0.698 & -0.024 & 2.257 \\
\hline $\mathrm{BA}\left(\mathrm{m}^{2}\right)$ & 20.470 & 0.272 & -0.047 & 1.441 \\
\hline Gross vol. $\left(\mathrm{m}^{3}\right)$, main stand & 58.978 & 0.851 & -0.024 & 2.445 \\
\hline \multirow[t]{2}{*}{ Gross merch. vol. $\left(\mathrm{m}^{3}\right)$} & 34.780 & 1.010 & -0.028 & 4.000 \\
\hline & \multicolumn{4}{|c|}{ Red pine } \\
\hline Height & 1.472 & 0.999 & -0.027 & 1.332 \\
\hline $\mathrm{BA}\left(\mathrm{m}^{2}\right)$ & 18.008 & 0.317 & -0.052 & 2.097 \\
\hline Gross vol. $\left(\mathrm{m}^{3}\right)$, main stand & 16.496 & 1.182 & -0.034 & 2.285 \\
\hline Gross merch. vol. $\left(\mathrm{m}^{3}\right)$ & 6.348 & 1.473 & -0.038 & 3.547 \\
\hline
\end{tabular}

